\title{
Searching for Alien Life Having Unearthly Biochemistry
}

\author{
Harry Jones \\ NASA Ames Research Center
}

Copyright (C) 2003 Society of Automotive Engineers, Inc.

\begin{abstract}
The search for alien life in the solar system should include exploring unearthlike environments for life having an unearthly biochemistry. We expect alien life to conform to the same basic chemical and ecological constraints as terrestrial life, since inorganic chemistry and the laws of ecosystems appear to be universal. Astrobiologists usually assume alien life will use familiar terrestrial biochemistry and therefore hope to find alien life by searching near water or by supplying hydrocarbons. The assumption that alien life is likely to be based on carbon and water is traditional and plausible. It justifies high priority for missions to search for alien life on Mars and Europa, but it unduly restricts the search for alien life. Terrestrial carbon-water biochemistry is not possible on most of the bodies of our solar system, but all alien life is not necessarily based on terrestrial biochemistry. If alien life has a separate origin from Earth life, and if can survive in an environment extremely different from Earth's, then alien life may have unearthly biochemistry. There may be other solvents than water that support alien life and other elements than carbon that form complex life enabling chain molecules. Rather than making the exploration-restricting assumption that all life requires carbon, water, and terrestrial biochemistry, we should make the exploration-friendly assumption that indigenous, environmentally adapted, alien life forms might flourish using unearthly biochemistry in many places in the solar system. Alien life might be found wherever there is free energy and a physical/chemical system capable of using that energy to build living structures. Alien life may be discovered by the detection of some general non-equilibrium chemistry rather than of terrestrial biochemistry. We should explore all the potential abodes of life in the solar system, including those where life based on terrestrial biochemistry can not exist.
\end{abstract}

\section{INTRODUCTION}

NASA's astrobiology program should include a search for exotic life forms having an unearthly biochemistry in our solar system and beyond. Exotic life forms may have biochemistry totally unlike that of Earth organisms. Alien life forms may be disturbingly different and nearly unrecognizable, or their independent origin may be revealed only by detailed biochemical analysis. Other solvents than water may support exotic life, and other elements than carbon may form life-enabling chain molecules. Exotic life forms may not be restricted to planets in the "habitable zone" defined by Earth-like conditions.

The assumption that all life must be Earth-like, based on carbon and water, motivates valuable research on extreme terrestrial life and helps prioritize planetary exploration targets, but it is too limiting. It suggests that life in our solar system may be restricted to Earth. We should instead make the exploration-friendly assumption that exotic life forms using different biochemistries might exist in unearthlike places in our solar system. Many bodies in our solar system may support environmentally adapted indigenous life. We should explore all the potential abodes of life, including those where carbon and water based life could not survive.

We do not know if the correct scientific hypothesis is that extraterrestrial life must be very similar to, or can be very different from, Earth life. The assumption that all life must be like life on Earth goes far beyond any conceivable factual basis. Given the immensity of the universe, the hundreds of billions of stars in each of the hundreds of billions of galaxies, any possible form of life must exist somewhere.

We can assume that alien life will be based on familiar chemical principles. We can assume that extraterrestrial ecosystems will follow the same ecological principles as the terrestrial biosphere. The best diagnostic for alien life will be the detection of an ecosystem or a biosphere. Alien life will probably be detected as some unanticipated exotic chemistry, not by the presence of terrestrial biochemistry.

\section{EXOBIOCHEMISTRY}

A previous paper described exobiochemistry, which is the possible biochemistry of extraterrestrial life (1). Exobiochemistry is a development of familiar chemical principles. Since the same atomic elements are found everywhere in the universe, any possible 
exobiochemistry must be based on well-known chemical and molecular reactions. Alien life will likely use the same energy sources as Earth life, including light, biologically produced organic material, and abiotic chemicals. It must use the same atomic elements and molecules found everywhere in the universe.

How does chemistry support life? Organisms obtain and control energy by using reduction-oxidation. (redox) chemical reactions that are based on electron transport. Terrestrial life has evolved a complex sequence of organic catalysts that help store and release energy in cells. Inorganic molecules found in the environment act as electron carriers to facilitate the transfer of electrons.

On Earth, photosynthesis is the most important energy capturing process and free oxygen is now the predominant electron buffer. In photosynthetic plants, energy is captured by the absorption of a photon of radiant light. Carbon dioxide and water are converted to organic material and oxygen. Animals ingest the carbohydrates produced by plants and use respiration to obtain energy. In the reverse of photosynthesis, respiration converts organic material and oxygen to carbon dioxide and water, releasing energy. $\mathrm{O}_{2} / \mathrm{H}_{2} \mathrm{O}$ (oxygen/water) is the redox (reduction-oxidation) couple used for electron transfer in plant photosynthesis and animal respiration. $\mathrm{S} / \mathrm{H}_{2} \mathrm{~S}$ (sulfur/hydrogen sulfide) is the redox couple used for photosynthesis by sulfur bacteria that existed before the Earth had an oxygen atmosphere. Many other inorganic redox couples are found on Earth and should occur on extraterrestrial bodies.

We expect extraterrestrial life, like terrestrial life, to capture, store, and release energy using reduction and oxidation (redox) reactions, probably using universally available inorganic redox couples. Energy can be captured by any process that reduces carbon (or some other suitable electron acceptor) by oxidizing hydrogen (or some other electron donor). Any inorganic redox couple such as oxygen and water or sulfur and hydrogen sulfide can be used as the electron buffer in a closed oxidation-reduction cycle.

All terrestrial life has a single origin and is based on hydrocarbons and water. All terrestrial life uses the same complex biochemistry with DNA, RNA, ATP, etc. Although it is very possible that extraterrestrial organisms will be based on carbon and water, some other biochemistry may be discovered on other planets. The possible exobiochemistry is constrained by the extraterrestrial environment, including temperature, availability of water, and atmospheric composition and pressure. Another atomic base could replace carbon. Some other solvent, perhaps ammonia, $\mathrm{NH}_{3}$, could replace water. It is reasonable to expect water-based biochemistry similar to Earth's in similar environments, but we should allow for the possibility of unearthly biochemistry. Terrestrial biochemistry provides a basis for generalization and extrapolation but it can not define the range of any possible exobiochemistry. Attempts to detect extraterrestrial ecology should not assume hydrocarbon chemistry, especially in unfavorable environments.

\section{CARBON AND WATER}

ARE CARBON AND WATER NECESSARY FOR LIFE? Carbon is so biolchemically suitable and so widely available that it is hard to imagine that any form of chemically based life could avoid using it. The same can be said for the other elements commonly used by terrestrial life, $\mathrm{H}, \mathrm{O}, \mathrm{N}, \mathrm{P}$, and $\mathrm{S}$ (hydrogen, oxygen, nitrogen, phosphorus, and sulfur). Although alien life will probably use carbon and the other biogenic elements, its exobiochemistry may be significantly different than that of terrestrial life.

The suitability and availability of water similarly suggest that life on other planets requires water. John Oro says "liquid water (is) commonly believed to be a prerequisite for biological activity," (2, p. 10), Christopher McKay states "(w)ithout liquid water, there is no prospect for the growth of life as we know it," (3, p. 69), and the National Research Council Space Studies Board indicates "(a)s a minimum for life to arise ... liquid water ... would appear to be required" $(4$, p. 56). Bruce Jakowsky suggests that the major requirements for life seem to be liquid water, a usable energy source, and the common elements used by life $(5, \mathrm{pp} .226,260)$. David Darling states that carbonbased molecules, liquid water, and a suitable energy source are generally considered necessary for life. $(6, p$. 14)

Donald Goldsmith and Tobias Owen present a detailed case that life is likely to be based on carbon and water:

"Life on Earth relies on carbon chemistry, and on water as a solvent, not by accident, but because carbon and water have an inherent superiority. The case for carbon may be stronger than that for water, but we should not feel narrow-minded in anticipating that most alien life forms rely on the same element and on the same solvent for their basic chemistry of life." (Italics in the original.) $(7, p$. 238)

The italicized "most" acknowledges that carbon and water are not inevitable. It seems that most exobiologists believe that life on other planets definitely or most likely is based on carbon and water, and that this assumption should guide the search for alien life.

Carl Sagan discussed alternatives to carbon and water and reached a different conclusion,

"(T)errestrial biochemistry may not be the only possible biochemistry or even the best possible 
biochemistry ... in searching for extraterrestrial life an open mind is a prime asset." $(8, p .86)$

OTHER SOLVENTS THAN WATER - Water has many properties that make it suitable for the chemistry of life. It is common, being a stable compound of two very abundant elements, hydrogen and oxygen. It is a liquid over a wide range of temperatures and acts as a good reservoir for heat. Water ice floats, insulating the water beneath it. Water is a good solvent, especially so because it is a polarized molecule with positive and negatively charged portions.

Water appears to be stable and inert on the surface of the Earth, but this is because water has always been present on Earth and has reacted with all possible substances. Water is damaging to DNA. Water is violently reactive on Mars. Water was present on Earth before life. Earth life is compatible with water and uses the properties of water because Earth life originated in water.

The most important properties of a solvent that might be used in exobiochemistry are that it be a simple compound of common elements so that it is relatively abundant, and that it dissolve a large enough number of compounds to form a self-organizing system. Many writers have suggested other solvents that could substitute for water. Candidate solvents include ammonia, hydrocyanic acid, and methane, which all have low freezing points. Ammonia and hydrocyanic acid are also good heat reservoirs and are polar molecules.

OTHER LINKING ELEMENTS THAN CARBON - Carbon forms many more compounds than any other element. But relatively few of the resulting organic molecules are used as the basic building blocks of life. Carbon is tetravalent and so can form single electron bonds with four other elements, but silicon is a frequently suggested alternative because it is also tetravalent. The idea that high temperature life might be based on silicon was first suggested by Reynolds in 1893. (6, p. 13)

Nitrogen and phosphorus usually form three bonds but can form four. Carbon can form long chains based on stable bonds to two adjacent carbons, with the two remaining bonds available for attached atoms or molecules. Other elements can also form chains, but they are less stable than carbon molecules at water temperatures. It seems only slightly more difficult to form long chains using alternating kinds of elements. Partial alternation occurs in the most important molecules used by terrestrial life. Proteins have $\mathrm{C}-\mathrm{C}-\mathrm{N}-\mathrm{C}-\mathrm{C}-\mathrm{N}$ and nucleic acids have C-C-C-O-P-O-C-C-C-O-P-O.

Because of the abundance and chemical properties of carbon, it will probably be used to some extent by any chemical life form. Just as on Earth, all the common reactive elements - $C, H, O, N, P$, and $S$ - will probably have an important biological role. But alien biochemistry may be very different from terrestrial biochemistry.

\section{WHAT SHOULD WE ASSUME ABOUT ALIEN LIFE?}

WHY ASSUME MOST LIFE IS EARTH-LIKE ? - Assuming that alien life is more or less Earth-like is a very useful assumption. A good scientific hypothesis leads to productive research and the expansion of knowledge, even if it has to be modified or abandoned later. The assumed similarity between terrestrial and extraterrestrial life inspires interesting research on terrestrial life. We try to grow terrestrial microbes under alien conditions. We investigate life in extreme terrestrial environments. We devise detectors for Earth-like life on Mars. The assumption that life is likely to be based on carbon and water helps us prioritize missions by focusing exploration on those rare places in the solar system where water may exist of have existed.

The assumption that life must be Earth-like is not necessarily correct. It is supported by analogies with terrestrial life and by hypotheses about how terrestrial life may have originated. But whatever happened on Earth, something else may have happened somewhere else. Fundamentally different kinds of life may possibly develop in exotic environments.

Decisions must be made using the knowledge available. We have to make assumptions in exploring space. But the carbon-water hypothesis is too narrow to guide the search for life. There are other solvents than water that may support life, and other elements than carbon that may form "organic" chains. The less restrictive assumption that life could be different in different environments suggests wider exploration.

WHY ASSUME LIFE MAY HAVE UNEARTHLY BIOCHEMISTRY? - The terrestrial analogy inspires us to search for potentially life-bearing planets in the habitable zone of other stars, but it is self-defeating in exploring our own solar system. An assumption that all life is Earth like implies that the habitable zone includes only the Earth and that no other solar system bodies can support life. Indeed, the astronomer Michael Hart was so impressed by the low early solar flux and the ice ages that he believes that the habitable zone is very small and that terrestrial life exists only by an improbable sequence of accidents (9 p. 26). And more recent suggestions of an early "snowball Earth" support this. The use of the terrestrial analogy is discouraging since it seems that searching for life in our solar system is doomed to failure.

We now know that Earth-like life can not exist on most of the bodies of the solar system. If life exists in these alien environments, it must be truly exotic. There is no possible way to prove that exotic life forms can not exist. Each solar system body should be explored for indigenous life in an objective manner.

Astrobiology should search for exotic life forms in our solar system. There are many more places to explore for 
exotic life in our solar system than for familiar carbonwater life. The discovery of exotic life forms would have a profound meaning for our concept of life. Merely considering the idea expands our thinking. We should make the exploration-friendly assumption that exotic life forms may exist and be discovered in our solar system.

WHAT CAN WE ASSUME ABOUT EXOTIC LIFE FORMS? - Extraterrestrial biochemistries may be very different from terrestrial biochemistry, but extraterrestrial ecologies will be strongly similar to terrestrial ecologies. Life on Earth has spread from a single origin, but it has adapted to an impressive range of environments. Earth's biosphere is a complex of different ecosystems, all functioning according to the same fundamental ecological principles. Extraterrestrial ecosystems can be expected to follow the same principles as terrestrial ecology.

The ecosystem analogy is stronger than the carbon and water analogy. There are many terrestrial ecosystems but only one terrestrial biochemistry. Terrestrial ecology is based on general dynamic systems principles while terrestrial carbon and water biology may be a unique result of environment and accident. The principles of dynamic systems are operative in electrical and mechanical systems, economics, and even politics. Our understanding of the principles of terrestrial ecosystems is more soundly based than our knowledge of the origin of terrestrial life.

Feinberg and Shapiro were pioneers of the ecological approach to extraterrestrial life. They consider that there are only three necessary conditions for life to develop; "(1) a flow of free energy, (2) a system of matter capable of interacting with the energy and using it to become ordered, and (3) enough time to build up the complexity of order that we associate with life" $(9, p .180)$.

The first and third conditions are met everywhere in the solar system. All of the planets and moons of our solar system have the same energy sources as the Earth solar radiation, impact accretion energy, and tidal energy - but the magnitudes of the energy flows differ greatly. And all the solar system bodies have had the same duration as the Earth. Since low temperature slows chemical reaction rates, the outer planets have had effectively less time for life to develop.

The second condition is the requirement for a supporting system of matter. We know that carbon and water chemistry can sustain life, but water is rare or absent on other bodies of the solar system. We should search for carbon and water life on Mars - at the edge of the ice cap, underground, and other places where water may exist, on Europa - on the ice cap and in the ocean under the ice cap, and even on Jupiter - in the clouds where temperatures and pressures are Earth-like. But supporting systems for life other than carbon and water chemistry may occur elsewhere.
Shapiro recently restated the minimal requirements for chemically based life forms as "an appropriate set of simple chemicals, an energy supply that interacted with them, and a liquid or dense gas medium." (10, p. 140) Jupiter, Saturn, and Titan have dense gas atmospheres and are interesting places to search for life. Life seems also possible in solids, such as in ice, near the surface of rocks, and even underground on Earth.

Exobiologists have become more willing to consider alien life having an unearthly biochemistry. David Darling moderates his carbon-water assumption to state:

"Just as uncovering one other example of carbon and water biology would suggest many more examples were waiting to be found, one case of life with a fundamentally different basis would show that our terrestrial notions of life were hopelessly parochial." (6, p. 178)

The National Research Council's Committee on the Origins and Evolution of Life has been considering the possibility of forms of life different from familiar terrestrial life.

"Life-related studies limited to a purely terrestrial biochemical perspective are unnecessarily constraining given the diversity of locales available for study in the next decade." (11)

Because extraterrestrial life will produce chemical indications of its existence, exobiochemistry can guide the search for alien life. Any significant departure from chemical equilibrium is evidence for exobiochemistry and alien life, but detecting an entire ecosystem with systematic related departures from the expected equilibrium would be more convincing.

\section{EXOECOLOGY}

Ecology describes the relations and interactions of living things with their environment. Ecosystems are highly constrained by their physical surroundings and by the principles of systems design. For example, all ecosystems conserve matter and energy and obey dynamic constraints. An ecosystem is the lowest level in the hierarchy of life that forms a complete, closed, sustainable system. This means that any extraterrestrial life must be part of an extraterrestrial ecosystem. The general principles of ecology can help guide the search for extraterrestrial life.

A previous paper developed the principles of extraterrestrial ecology, or exoecology (12). Twenty basic principles of exoecology were summarized under the five areas of environmental interaction, ecosystem design, ecosystem dynamics, material cycling, and temporal cycles. 


\section{A. ENVIRONMENTAL INTERACTION:}

1. The exoenvironment will limit the potential types of exoecology.

2. Different exoenvironments will support different exoecosystems.

3. The exoecology will be adapted to its environment by evolution.

4. The environment will be affected by any exoecology.

B. ECOSYSTEM DESIGN:

1. An exoecosystem will require energy input and use material recycling.

2. An exoecosystem will contain a trophic food web of producers, decomposers, and possibly primary and secondary consumers.

3. The number of trophic levels will be four or fewer.

4. An exoecosystem will have a hierarchy of compartmentalized subsystems.

\section{ECOSYSTEM DYNAMICS:}

1. The energy flux and storage will be an order of magnitude lower for each higher level of the trophic pyramid.

2. Higher energy flow between trophic levels will increase stored energy and exoecosystem diversity.

3. Energy will be dissipated and converted to heat.

4. Energy is not necessarily the limiting factor in exoecosystem growth.

\section{MATERIAL CYCLING:}

1. The material cycle will be closed by decomposers.

2. Material cycles with a gaseous phase will cause uniform distribution of the material.

3. Material cycles with no gaseous phase will have a more varied spatial distribution of the material.

4. Material availability may be the limiting factor in exoecosystem growth.

\section{E. TEMPORAL CYCLES:}

1. Temporal cycles in exoecosystems can be caused by external or internal factors.

2. External astronomical factors may cause day and night, tides, and seasons.

3. Internal dynamics will cause exoecological succession.

4. Stable systems typically have only one limiting factor.

The principles of exoecology are more fundamental and inevitable, more extensive and predictive than any so far established for exobiology. We know much more about the behavior, structure, and effects of an exoecosystem than of exobiology. We should search for exoecosystems based on the general principles of exoecology, rather than trying to detect exobiology analogous to terrestrial biology.

\section{HOW CAN WE SEARCH FOR EXOTIC LIFE FORMS?}

Once we accept the possibility of exotic life forms not based on carbon and water, what exploration program do we follow? The key is the ecological assumption that the principles governing Earth's ecosystems and other dynamic systems also apply to alien ecologies.

The suggested search assumptions are as follows:

- Exotic life forms may exist wherever there is free energy and a suitable physical/chemical basis. That is, almost anywhere.

- The energy supporting alien life could be sun light, volcanic, or chemical, as on Earth. The rate of activity would probably be proportional to the energy flow and slower on colder bodies.

- Life is not necessarily based on carbon and water chemistry as it is on Earth. Alien life may use some other solvent or additional linking elements.

- The distinguishing characteristic of alien life will be the formation of ecosystems and a biosphere.

Probably only a small part of any biosphere will be living material, but much or all of the material may be cycled by life. The biosphere will increase the abundance of certain types of molecules in a way distinguishable from chemical equilibrium. For instance, there may be combinations of gases that can not exist in equilibrium, such as the oxygen and methane that coexist in the Earth's atmosphere. Alien life and ecology will probably be best detected by distinctive chemical abundances, not by the presence of any particular life substance. Confirmation will be based on the recognition of systems exemplifying some emergent order, showing the higher level of system complexity characteristic of ecosystems and organisms far from thermodynamic equilibrium.

It is time to begin the search for exotic life forms. The search should include chemical experiments, ecological simulations, Hubble observations, development of detection instruments for planetary orbiters and landers, and robotic and manned missions to solar system bodies.

\section{CONCLUSION}

The most important purpose of exploring the solar system is to search for life. We can not assume all life will be based on carbon and water biochemistry.

We should explore all the potential abodes of life in the solar system, giving priority to those that could support carbon and water based life but excluding none. A reasonable sequence of targets would be Mars again, then the Galilean satellites of Jupiter (Europa, lo, Ganymede, Callisto) which may have water, then Jupiter, Saturn, and Titan, which may support exotic life. What we specifically suggest, for a Europa lander or any mission, 
is a search for exotic life forms as well as carbon and water based life.

For each planetary body, we should establish the physical and chemical limits to life, and then attempt to detect the environmental effects of ecosystems by studying chemical abundances and equilibrium. Only after detection of an environmental disequilibrium can we begin to guess the chemical basis of any alien life.

\section{REFERENCES}

1. Jones, Harry, "Exobiochemistry and the Search for Alien Life," Proceedings of the 32nd International Conference on Environmental Systems, ICES 200201-2472, 2002.

2. Oro, J., S. W. Squyres, R. T. Reynolds, and T. M. Mills, "Europa: Prospects for an Ocean and Exobiological Implications," in Exobiology in Solar System Exploration, ed. by G. Carle, D. Schwartz, and J. Huntington, NASA SP 512, 1992.

3. McKay, C. P., "Mars: A Reassessment of its Interest to Biology," in Exobiology in Solar System Exploration, ed. by G. Carle, D. Schwartz, and J. Huntington, NASA SP 512, 1992.

4. Space Studies Board, National Research Council, The Search for Life's Origins, National Academy Press, Washington, D. C., 1990.

5. Jakowsky, Bruce, The Search for Life on Other Planets, Cambridge University, 1998.
6. Darling, David, Life Everywhere: The Maverick Science of Astrobiology, Basic Books, New York, 2001.

7. Goldsmith, D., and T. Owen, The Search for Life in the Universe, Addison-Wesley, Reading, Mass, 1992.

8. Sagan, C., "The Solar System as an Abode of Life," in Biology and the Exploration of Mars, ed. by C. S. Pittendrigh, W. Vishniac, and J. P. T. Pearman, National Academy of Sciences, National Research Council, Washington, 1966.

9. Feinberg, Gerald, and Robert Shapiro, Life Beyond Earth, William Morrow, New York, 1980.

10. Shapiro, Robert, Planetary Dreams: The Quest to Discover Life beyond Earth, Wiley, New York, 1999.

11. National Academy of Sciences, press release, May 10, 2002.

12. Jones, Harry, "Extraterrestrial Ecology (Exoecology)," Proceedings of the 31st International Conference on Environmental Systems, ICES 200101-2143, 2001.

\section{CONTACT}

Harry Jones, Ph.D.

Mail Stop 239-8

NASA Ames Research Center

Moffett Field, CA 94035-1000

Phone: 650-604-5518

e-mail: Harry.W.Jones@nasa.gov 